Scand. J. of Economics 120(1), 159-182, 2018

DOI: $10.1111 /$ sjoe. 12207

\title{
Reference Pricing with Elastic Demand for Pharmaceuticals*
}

\author{
Ricardo Gonçalves ${ }^{\dagger}$ \\ Universidade Católica Portuguesa, 4169-005 Porto, Portugal \\ rgoncalves@porto.ucp.pt \\ Vasco Rodrigues \\ Universidade Católica Portuguesa, 4169-005 Porto, Portugal \\ vrodrigues@porto.ucp.pt
}

\begin{abstract}
In this paper, we re-examine the properties of two commonly adopted government reimbursement schemes for pharmaceuticals: reference pricing and fixed percentage reimbursement. We depart from the previous literature by assuming that the individual demand is price-sensitive and depends on the copayment rate (i.e., the part paid by each consumer). We obtain two novel results under reference pricing: first, as the copayment rate increases, so do pharmaceutical prices; second, this increase in pharmaceutical prices reduces social welfare. Whilst reference pricing does emerge as a preferable reimbursement scheme, demand elasticities and the copayment rate interact in complex ways. This leads (unexpectedly) to the possibility that a higher copayment rate (lower reimbursement rate) results in higher government expenditure.
\end{abstract}

Keywords: Copayment rates; pharmaceutical reimbursement schemes

JEL classification: I18; L13

\section{Introduction}

Purchasing pharmaceuticals is different from purchasing other goods: demand is often relatively inelastic and, in a large number of countries, individuals only pay a fraction of the price of the prescription pharmaceuticals they consume, whilst the government (or other third-party payers) are responsible for the remainder. Two widely adopted schemes to determine their relative contributions are fixed percentage reimbursement (FPR) and reference pricing (RP). Under FPR, the government pays a fixed

\footnotetext{
${ }^{\dagger}$ Both authors are affiliated with Católica Porto Business School and CEGE.

* Financial support from Fundação Ciência e Tecnologia (projects PTDC/EGEECO/100296/2008 and PEst-OE/EGE/UI0731/2011) is gratefully acknowledged. We thank Hélder Vasconcelos, Ricardo Ribeiro, Odd Rune Straume, two referees, and participants of the 2012 European Conference in Health Economics (Zurich), 6th Portuguese Economic Journal Meeting (2012, Porto), and 13th Portuguese Health Economics Conference (2013, Braga) for helpful comments and suggestions.

(C) The editors of The Scandinavian Journal of Economics 2016.
} 
percentage of the pharmaceutical's price and the patient is responsible for the remainder (also known as the copayment rate). By contrast, under RP, within each cluster of pharmaceuticals, ${ }^{1}$ one is chosen as the "reference" and the government only pays a fixed fraction of the "reference" pharmaceutical's price, even if the consumer buys a more expensive alternative. $^{2}$

In the last decades, RP has become a widely used scheme for price and (public) expenditure control in pharmaceutical markets. It has attracted significant attention in the literature, and a large consensus has emerged regarding its merits. Most models in the literature on RP share two common features: first, they assume pharmaceuticals are differentiated; second, they typically make the assumption of unit demand (i.e., each consumer is assumed to purchase exactly one unit of a pharmaceutical variety or none). Under the assumption of unit demand with full market coverage (e.g., Brekke et al., 2007), it should not be surprising that social welfare is equal across reimbursement regimes as there are no quantity effects. Reimbursement regimes merely determine who captures the surplus created, but not its amount.

In this paper, our main aim is to analyze the impact of considering that each individual consumer's demand depends on prices - a clear departure from the previous literature, which commonly assumes individual unit demand - under reimbursement schemes such as FPR and RP, in a full market coverage setting with differentiated pharmaceuticals. This strikes us as a realistic assumption, at least for some health conditions. For instance, in the treatment of health conditions that result in recurring episodes of symptoms of variable intensity, such as sleeping disorders, muscular pain, and migraines, pharmaceuticals might be prescribed during a significant period of time (e.g., under longterm prescriptions). It is entirely plausible that the patient might react to the price of the prescribed pharmaceutical when deciding whether to take it on a specific occasion. ${ }^{3}$ Even in the treatment of chronic diseases and conditions, such as hypertension, high cholesterol, and asthma, treatment adherence has been shown to depend on patients'

\footnotetext{
${ }^{1}$ Several criteria could be used to cluster pharmaceuticals: chemical, pharmacological, and therapeutic (Miraldo, 2009).

${ }^{2}$ Naturally, RP is only viable if more than one pharmaceutical is available for the treatment of a condition, and it is especially used when low-price generic competition exists. For more detailed reviews of RP, see López-Casasnovas and Puig-Junoy (2000) and Galizzi et al. (2011).

${ }^{3}$ It is important to note that the most obvious candidate to satisfy this assumption is the over-the-counter (OTC) market for pharmaceuticals. However, OTC pharmaceuticals are not subject to prescription and, hence, typically fall outside the scope of reimbursement schemes.
}

(c) The editors of The Scandinavian Journal of Economics 2016. 
copayments. ${ }^{4}$ The health conditions and associated pharmaceuticals likely to satisfy our assumption are a non-negligible portion of the pharmaceutical market. For instance, a statin (Pfizer's Lipitor), which is used for the treatment of high cholesterol, was the top selling drug in the United States in 2011 (the year its patent expired), with overall sales close to nine billion dollars ( 3 percent of total pharmaceutical expenditure), and Gibson et al. (2006) report that statin treatment adherence depends negatively on copayment rates. ${ }^{5}$ However, our setting might be less adequate for pharmaceuticals that are used to treat serious non-recurring conditions, in which case the patient will often be limited to the choices of either following his physician's prescription and buying the pharmaceutical, or not buying it at all. In this case, the framework used by Brekke et al. (2007) seems more fitting.

We obtain two novel results. First, we find that, under RP, prices increase with the copayment rate, converging to FPR prices as it approaches one. This differs from Brekke et al. (2007) and Gonçalves et al. (2015), who find that, under RP, prices are independent of the copayment rate. ${ }^{6}$ Second, we find that whilst social welfare under RP is always higher than under FPR (converging to the latter as the copayment rate approaches one), it decreases with the copayment rate.

The impact of the elastic demand assumption on RP explains the differences from the results in the previous literature. In summary, whereas under FPR an increase in the copayment rate (assuming all else constant) has no impact on the relative prices faced by the consumer, because all prices rise by the same percentage, this is not so under RP - an increase in the copayment rate changes the relative prices in favor of the more expensive (branded) pharmaceutical and, consequently, increases its market share. In the face of this, the branded producer takes the opportunity to raise prices. In equilibrium, the generic price also increases and, thus, higher copayment rates lead to higher prices.

From the point of view of social welfare, under RP, higher copayment rates lead to higher prices, lower consumption levels, and higher consumer pharmaceutical expenditure. In addition, as the copayment rate increases, a lower fraction of consumers purchase the branded pharmaceutical because (in equilibrium) its effective price increases relatively more than the

\footnotetext{
${ }^{4}$ Treatment adherence refers to patients taking their medication as prescribed - quantity and frequency. See Lexchin and Grootendorst (2004), Tseng et al. (2004), Gibson et al. (2005), Goldman et al. (2007), and Eaddy et al. (2012), among others.

${ }^{5}$ Another statin, Crestor, ranked seventh, with a total expenditure of close to five billion dollars in 2011 (information retrieved from the Medical Expenditure Panel Survey http:// meps.ahrq.gov/mepsweb/data_stats/summ_tables/hc/drugs/2011/hodrugest_totexp2011.shtml).

${ }^{6}$ In Brekke et al. (2007), this is true only for therapeutical reference pricing - the setting that is directly comparable to ours - but not for generic reference pricing.
} 
generic. These two factors contribute to a reduction in social welfare. This is in stark contrast with the case of inelastic demand, where an increase in the copayment rate is effectively a welfare-neutral transfer between the government and consumers (the former spends less in pharmaceutical reimbursement, to the detriment of the latter who must now bear a higher copayment).

From the point of view of policy, two main conclusions emerge. First, from a social welfare perspective, RP is preferable to FPR. Second, under $\mathrm{RP}$, an increase in the copayment rate might not necessarily lead to a decrease in government expenditure - for low copayment rates and high elasticities, an increase in the copayment rate leads to a price rise that more than compensates the decrease in the quantity consumed and in the reimbursement rate.

Our results are driven by the combined assumptions of vertical differentiation between branded and generic pharmaceuticals and elastic demand at the individual consumer's level. Vertical differentiation is not unusual in models of the pharmaceuticals market (e.g., Merino-Castelló, 2003; Brekke et al., 2011), although some authors use horizontal differentiation (e.g., Miraldo, 2009) and others combine horizontal and vertical differentiation (e.g., Brekke et al., 2007). By contrast, the elastic demand of an individual consumer is novel: the extant literature assumes unit demand at the individual consumer level, even if this could result in elastic aggregate demand when the assumption of full market coverage is relaxed (e.g., Merino-Castelló, 2003; Miraldo, 2009; Brekke et al., 2011). In spite of these differences, our model confirms that RP is associated with enhanced price competition, as also shown by Merino-Castelló (2003) and Brekke et al. (2007), among others. ${ }^{7}$

The paper has the following structure. In Section II, we describe the model. In Sections III and IV, we discuss our results and their welfare implications, respectively. We conclude in Section V. An Appendix contains a detailed derivation of our results and additional useful graphics. An Online Appendix is available as supporting material.

\section{Model}

Our model is loosely inspired by Rath and Zhao (2001) and Gu and Wenzel (2009). Consumers are assumed to buy a Hicksian composite good (denoted by $h$ ) and a differentiated pharmaceutical that exists in two varieties - branded $(b)$ and generic $(g)$ - each of which is produced

\footnotetext{
${ }^{7}$ In addition, Brekke et al. $(2009,2011)$ present empirical evidence of significant price reductions for all pharmaceuticals following the introduction of RP in Norway.
}

(c) The editors of The Scandinavian Journal of Economics 2016. 
by a different firm. We further assume that these two firms compete by simultaneously setting prices.

As in Hanemann (1984), consumers' decisions involve both a qualitative or discrete choice ("which pharmaceutical variety, branded or generic, to consume") as well as a continuous choice ("how many units of the chosen pharmaceutical variety to consume"). Suppose that a consumer of type $c$ has decided to consume only variety $i \in\{b, g\}$. Given this decision, as in $\mathrm{Gu}$ and Wenzel (2009), we assume that his conditional direct utility from consuming $q_{h}$ units of the Hicksian good and $q_{i}$ units of the differentiated pharmaceutical is

$$
\bar{u}\left(q_{h}, q_{i}, c\right)=q_{h}-\frac{\varepsilon}{1-\varepsilon} q_{i}^{(\varepsilon-1) / \varepsilon}-f_{i}(c),
$$

where $f_{i}(c)$ is a consumer-specific disutility parameter discussed in more detail below. This utility function leads to isoelastic demand functions for the differentiated pharmaceutical, where the (absolute value of the) demand elasticity is equal to $\varepsilon$. Note that the conditional direct utility function increases with the consumption of the differentiated pharmaceutical, as increased consumption reduces a negative element (assuming $\varepsilon<1$ ) that can be interpreted as "disease" or "discomfort". 8

We also assume that consumers differ in the degree to which they distrust the generic pharmaceutical to be therapeutically equivalent to the branded product. This seems plausible not only among the general public but also among trained physicians. Kesselheim et al. (2008, p. 2514) note that generics "[...] may differ in peripheral features, such as pill color or shape, inert binders and fillers, and the specific manufacturing process". However, "[s]ome physicians and patients have expressed concern that bioequivalent generic and brand-name drugs may not be equivalent in their effects on various clinical parameters, including physiological measures such as heart rate or blood pressure, important laboratory measurements, and outcomes such as health system utilization or mortality" (Kesselheim et al., 2008, p. 2515). The scientific debate on whether bioequivalence translates on to treatment effect equivalence is still ongoing, but at the very least suggests that it is plausible and possibly rational for a consumer to suspect that a generic drug might not have the same treatment effect as the branded drug. ${ }^{9}$ In this context, parameter $f_{i}(c)$ in the conditional

\footnotetext{
${ }^{8}$ In addition, the marginal utility of consumption of the chosen differentiated pharmaceutical is decreasing (i.e., increasing consumption levels contribute gradually less towards the treatment of the underlying health condition).

${ }^{9}$ Kesselheim et al. (2008) conclude, in their meta-analysis of various studies, that generics and branded drugs have similar clinical outcomes in the treatment of cardiovascular diseases; by contrast, Borgherini (2003) reaches an opposite conclusion for psychoactive drugs. Kobayashi et al. (2011) discuss evidence from various countries (United States, Spain, Portugal, Germany,
} 
utility function measures the utility loss consumers suffer by buying a drug they trust less than the branded pharmaceutical. By definition, $f_{b}(c)=0$, as the branded pharmaceutical is the benchmark against which trust is measured. As for consumers who buy the generic drug, we assume $f_{g}(c)=t c$. The parameter $c \in[0,1]$ measures the "level of distrust" and is consumer-specific - effectively the consumer type - and we assume it to be uniformly distributed in the $[0,1]$ interval. ${ }^{10}$ The parameter $t>0$ measures the negative impact of this distrust on the consumer's conditional utility.

Maximizing the conditional direct utility subject to the consumer's budget constraint $\hat{p}_{i} q_{i}+p_{h} q_{h}=m$ (where $\hat{p}_{i}$ and $p_{h}$ represent the prices of the differentiated pharmaceutical and Hicksian good, respectively, and $m$ represents the consumer's income), and normalizing $p_{h}=1$, the associated conditional demand functions are given by

$$
\begin{aligned}
& \bar{q}_{h}\left(\hat{p}_{i}, m\right)=m-\hat{p}_{i}^{1-\varepsilon} ; \\
& \bar{q}_{i}\left(\hat{p}_{i}, m\right)=\frac{1}{\hat{p}_{i}^{\varepsilon}} .
\end{aligned}
$$

In this context, consumer $c \in[0,1]$ obtains the following conditional indirect utility from consuming pharmaceutical variety $i$ :

$$
\bar{v}_{i}\left(\hat{p}_{i}, m, c\right)=m-\left(\frac{1}{1-\varepsilon}\right) \hat{p}_{i}^{1-\varepsilon}-f_{i}(c) .
$$

We implicitly assume that each type of consumer purchases branded or generic pharmaceuticals, but not both simultaneously; in effect, consumers compare the maximum conditional utility they can obtain (given their budget constraint) by consuming (a certain quantity of) each pharmaceutical variety, and they choose whichever yields the highest conditional utility.

Both the choice between the generic and branded products as well as the quantities consumed depend on the prices charged to the consumer,

and Norway) where consumers were (or are) skeptical about the effectiveness and safety of generic drugs. In a study on the underlying reasons for generic underuse in Portugal, Quintal and Mendes (2011) find that over 40 percent of respondents justify it because of their "lack of trust" in generics.

${ }^{10}$ Intuitively, we can interpret the model as one of vertical differentiation: the $[0,1]$ interval is a scale that measures the subjective perceived vertical differentiation between the branded pharmaceutical and the generic. Note also that we assume this disutility to be independent of the quantity consumed (i.e., lump sum), in a similar way to Gu and Wenzel (2009). In effect, this is equivalent to assuming a (fixed) consumer-specific distrust in the generic treatment effectiveness, which does not depend on the quantity the consumer decides to consume, and which only affects the discrete choice of the drug variety to purchase (branded or generic).

(c) The editors of The Scandinavian Journal of Economics 2016. 
and these depend on the reimbursement scheme in place. We analyze two reimbursement schemes: an FPR scheme (henceforth represented by the superscript " $F$ "), in which consumers pay the same percentage (copayment rate), $\theta \in[0,1]$, of their desired product's headline price (the headline price being the price set by the producer); and an RP scheme (henceforth represented by the superscript " $R$ "), where the consumer's copayment is based on the price of the reference pharmaceutical, which we assume to be the generic because it is cheaper and hence leads to a lower reimbursement expenditure. Under RP, consumers must pay a percentage (copayment rate) of the reference pharmaceutical's price if they choose to purchase it, but if they choose to purchase the branded pharmaceutical they must pay in full the price difference with respect to the reference price.

Under FPR, effective prices (i.e., the prices effectively paid by the consumer) are given by

$$
\hat{p}_{i}^{F}=p_{i}^{F}-(1-\theta) p_{i}^{F}=\theta p_{i}^{F}, \quad i=b, g,
$$

where we define $p_{i}^{F}, i \in\{b, g\}$, to be the headline prices set by firms under FPR. By contrast, under RP (assuming the copayment rate $\theta$ is similar across schemes), effective prices are given by

$$
\begin{aligned}
\hat{p}_{b}^{R} & =p_{b}^{R}-(1-\theta) p_{g}^{R}, \\
\hat{p}_{g}^{R} & =p_{g}^{R}-(1-\theta) p_{g}^{R}=\theta p_{g}^{R},
\end{aligned}
$$

where $p_{i}^{R}, i \in\{b, g\}$, are the headline prices set by firms when the RP scheme is in place.

We also refer to the no reimbursement (NR) scenario, where patients must support the headline prices in full; this is equivalent to setting $\theta=1$ in equations (3) or (4). ${ }^{11}$ Finally, production costs are assumed to be zero for both producers. ${ }^{12}$

\footnotetext{
${ }^{11}$ This would generally be the relevant scenario for OTC pharmaceuticals.

${ }^{12}$ Brekke et al. (2007) and Miraldo (2009) also assume zero marginal production costs, whilst Brekke et al. (2011) assume a positive marginal production cost. We make the assumption that production costs are negligible and equal across producers, as there is no reason to assume a cost advantage in favor of any of them. As suggested by an anonymous referee, we have solved the model under positive production costs. Unfortunately, this introduces significant complexity in the analysis, and there are no closed-form solutions under both FPR and RP. Nevertheless, we have conducted several simulations, which indicate that all our results hold for a sufficiently small production cost.
} 


\section{Pricing}

\section{Fixed Percentage Reimbursement}

Under FPR, the (Nash) equilibrium headline prices are given by ${ }^{13}$

$$
\begin{aligned}
& p_{b}^{F}=\left[\frac{2(1-\varepsilon) t}{3 \theta^{1-\varepsilon}}\right]^{1 /(1-\varepsilon)} ; \\
& p_{g}^{F}=\left[\frac{(1-\varepsilon) t}{3 \theta^{1-\varepsilon}}\right]^{1 /(1-\varepsilon)} .
\end{aligned}
$$

These results are in line with those of Brekke et al. (2007), Rodrigues et al. (2014), and Gonçalves et al. (2015). ${ }^{14}$ Note that $p_{i}^{F}=(1 / \theta) p_{i}^{N R}$, with $i \in\{b, g\}$. Under FPR, firms mark up their headline prices in an inversely proportional manner relative to the copayment rate; that is, the higher the copayment rate, the lower the mark-up is. Thus, headline prices, $p_{b}^{F}$ and $p_{g}^{F}$, are lowest when the patient's copayment rate is maximal $(\theta=1)$. Effective prices are equal in both cases and therefore equilibrium quantities are also the same: $\hat{p}_{i}^{F}=\hat{p}_{i}^{N R}(i \in\{b, g\})$. Under FPR, an increase in the copayment rate $\theta$ would lead, ceteris paribus (i.e., assuming no change in headline prices), to an increase in effective prices, therefore reducing the quantity consumed of both the branded and generic pharmaceuticals. In this context, both firms find it profit-maximizing to reduce headline prices and, thus, to reduce the (negative) impact on demand. It turns out that, in equilibrium, this reduction is sufficient for effective prices not to change and, thus, for individually consumed quantities and overall sold quantities of both goods to remain constant.

Inevitably, this hurts the profits of both firms. As mentioned earlier, this result is similar to that of Brekke et al. (2007) - in the case of no reference pricing, which is equivalent to our FPR setting. An increase in the copayment rate leads to a reduction in total pharmaceutical expenditure (which is equal to profits) and, consequently, to a reduction in government expenditure, but consumer surplus remains unchanged. ${ }^{15}$ From a different point of view, reimbursement through FPR can be seen as a public subsidy that ultimately benefits firms.

\footnotetext{
${ }^{13}$ More detail regarding the derivation of these results is provided in the Appendix.

${ }^{14}$ In particular, when $\varepsilon=0$ (inelastic demand), $\left.p_{b}^{F}\right|_{\varepsilon=0}=2 t / 3 \theta,\left.p_{g}^{F}\right|_{\varepsilon=0}=t / 3 \theta$, and the individual quantity purchased by each consumer is equal to one unit.

${ }^{15}$ Profit levels (which are equal to total pharmaceutical expenditure) thus become $\Pi_{i}^{F}=$ $(1 / \theta) \Pi_{i}^{N R}, i \in\{b, g\}$ (i.e., firms also increase their profits in an inversely proportional manner relative to the copayment rate). Also, note that consumer surplus $C S$ does not depend on $\theta$; as outlined above, the copayment rate has no impact on the effective prices that consumers must face, $\hat{p}_{b}^{F}$ and $\hat{p}_{g}^{F}$. Hence, the existence of an FPR scheme (through parameter $\theta$ ) does not affect consumer surplus (i.e., $C S^{F}=C S^{N R}$ ).
} 


\section{Reference Pricing}

Under RP, effective prices are given by equation (4). As the Appendix shows in more detail, the profit maximization's first-order conditions cannot be solved analytically and, hence, no closed-form solution exists for equilibrium prices, except when $\varepsilon=1 / 2$. Therefore, we have adopted the following strategy. First, we solve the model and obtain equilibrium prices for $\varepsilon=1 / 2$. Then, we use simulations to confirm the robustness of our results for different values of $\varepsilon$ (see the Online Appendix).

When $\varepsilon=1 / 2$, the (Nash) equilibrium headline prices are given by

$$
\begin{aligned}
& \left.p_{b}^{R}\right|_{\varepsilon=1 / 2}=\frac{2 \theta\left(1+\theta^{1 / 2}\right)}{4\left(1+2 \theta^{1 / 2}\right)^{2}} t^{2}, \\
& \left.p_{g}^{R}\right|_{\varepsilon=1 / 2}=\frac{\theta}{4\left(1+2 \theta^{1 / 2}\right)^{2}} t^{2} .
\end{aligned}
$$

It is particularly relevant to highlight the following (novel) result (we defer a more careful interpretation of the underlying rationale to the end of this section).

Proposition 1. With $\varepsilon=1 / 2$ under an RP scheme, both headline and effective equilibrium prices increase with the copayment rate.

Proof: In equilibrium, under an RP scheme with $\varepsilon=1 / 2$, headline prices are given by equation (A18), whose derivative with respect to $\theta$ is

$$
\begin{aligned}
& \frac{\left.\partial p_{b}^{R}\right|_{\varepsilon=1 / 2}}{\partial \theta}=\frac{\left(3 \theta^{1 / 2}+2 \theta+2\right) t^{2}}{4\left(1+2 \theta^{1 / 2}\right)^{3}} ; \\
& \frac{\left.\partial p_{g}^{R}\right|_{\varepsilon=1 / 2}}{\partial \theta}=\frac{t^{2}}{4\left(1+2 \theta^{1 / 2}\right)^{3}} .
\end{aligned}
$$

Both derivatives are positive for any $t>0$ and $\theta \in[0,1]$. Effective equilibrium prices are easily calculated using equations (4) and (6), yielding $\left.\hat{p}_{b}^{R}\right|_{\varepsilon=1 / 2}=\theta\left(1+\theta^{1 / 2}\right)^{2} t^{2} / 4\left(1+2 \theta^{1 / 2}\right)^{2}$ and $\left.\hat{p}_{g}^{R}\right|_{\varepsilon=1 / 2}=\theta^{2} t^{2} / 4\left(1+2 \theta^{1 / 2}\right)^{2}$. The derivative of effective prices with respect to $\theta$ is

$$
\begin{aligned}
& \frac{\left.\partial \hat{p}_{b}^{R}\right|_{\varepsilon=1 / 2}}{\partial \theta}=\frac{\left(1+3 \theta^{1 / 2}+4 \theta+2 \theta^{3 / 2}\right) t^{2}}{4\left(1+2 \theta^{1 / 2}\right)^{3}} ; \\
& \frac{\left.\partial \hat{p}_{g}^{R}\right|_{\varepsilon=1 / 2}}{\partial \theta}=\frac{\left(\theta+\theta^{3 / 2}\right) t^{2}}{2\left(1+2 \theta^{1 / 2}\right)^{3}} .
\end{aligned}
$$

Again, both derivatives are positive for any $t>0$ and $\theta \in[0,1]$.

When we compare these equilibrium prices with those obtained under an FPR scheme, we also obtain the following result. 
Proposition 2. With $\varepsilon=1 / 2$, both headline and effective equilibrium prices under an $R P$ scheme are never higher than under an FPR scheme.

Proof: RP equilibrium prices with $\varepsilon=1 / 2$ are given by equation (6). By evaluating FPR equilibrium prices (equation (5)) when $\varepsilon=1 / 2$, we obtain

$$
\begin{aligned}
& \left.p_{b}^{F}\right|_{\varepsilon=1 / 2}=\frac{4}{36 \theta} t^{2} ; \\
& \left.p_{g}^{F}\right|_{\left.\right|_{\varepsilon=1 / 2}}=\frac{1}{36 \theta} t^{2} .
\end{aligned}
$$

It is easily shown that $\left.p_{i}^{F}\right|_{\varepsilon=1 / 2}>\left.p_{i}^{R}\right|_{\varepsilon=1 / 2}$ for any $\theta \in[0,1)$ and $\left.p_{i}^{F}\right|_{\varepsilon=1 / 2}=$ $\left.p_{i}^{R}\right|_{\varepsilon=1 / 2}$ when $\theta=1, \quad i \in\{b, g\}$.

Effective equilibrium prices under FPR (when $\varepsilon=1 / 2$ ) are easily calculated using equations (3) and (5): $\left.\hat{p}_{b}^{F}\right|_{\varepsilon=1 / 2}=4 t^{2} / 9$ and $\left.\hat{p}_{g}^{F}\right|_{\varepsilon=1 / 2}=t^{2} / 9$. Effective equilibrium prices under RP (when $\varepsilon=1 / 2$ ) are given above (see the proof of Proposition 1). It is now straightforward to show that $\left.\hat{p}_{i}^{F}\right|_{\varepsilon=1 / 2}>\left.\hat{p}_{i}^{R}\right|_{\varepsilon=1 / 2}$ for any $\theta \in[0,1)$ and $\left.\hat{p}_{i}^{F}\right|_{\varepsilon=1 / 2}=\left.\hat{p}_{i}^{R}\right|_{\varepsilon=1 / 2}$ when $\theta=1$, $i \in\{b, g\}$.

Proposition 1 shows that headline prices under RP increase with $\theta$. This contrasts with the case of $\varepsilon=0$ (inelastic demand), where headline prices do not depend on $\theta:\left.p_{b}^{R}\right|_{\varepsilon=0}=2 t / 3$ and $\left.p_{g}^{R}\right|_{\varepsilon=0}=t / 3$ (effective prices, however, increase with $\theta$ ). When $\varepsilon>0$, an increase in $\theta$ (ceteris paribus) leads to an increase in both effective prices by the same amount. However, because it is lower in absolute terms, the effective price of the generic increases by more than the effective price of the branded pharmaceutical in percentage terms. The branded product becomes relatively less expensive, thus attracting some of the consumers that were previously buying the generic. That is, a lower fraction $c^{R}$ of consumers will purchase the generic - and a higher fraction will purchase the branded pharmaceutical (see equation (A12) in the Appendix). Therefore, although an increase in $\theta$ reduces the quantity demanded of both the branded and generic pharmaceuticals by each individual consumer, it leads to a decrease (increase) in the overall quantity demanded of the generic (branded) pharmaceutical because some consumers switch from the generic to the branded pharmaceutical (see equation (A21) in the Appendix). In this context, the branded producer finds it profit-maximizing to respond to the increase in the copayment rate with an increase in its headline price. In equilibrium, the generic producer does the same, and the increase in the copayment rate - coupled with the increase in headline prices - unequivocally increases effective prices and reduces 
individually consumed quantities, as well as overall demand for both pharmaceuticals. $^{16}$

Headline prices under FPR decrease with $\theta$ whilst under an RP scheme they increase, but the latter are always lower than (or equal to, when $\theta=1$ ) the former for a given value of $\varepsilon$, as suggested by Proposition 2 (in the case of $\varepsilon=1 / 2) \cdot{ }^{17}$ Because headline and effective prices are higher under FPR (except for $\theta=1$, in which case they are equal), this suggests that RP is more effective in bringing about price competition than FPR, to the benefit of consumers. This result is in line with that of the existing literature (Merino-Castelló, 2003; Brekke et al., 2007, 2011). In particular, as pointed out by Brekke et al. (2011), compared to FPR and for given prices, RP makes the branded pharmaceutical more expensive for consumers (as the part of the price that is subject to reimbursement is lower). The generic producer enhances this effect by lowering its headline prices and, thus, the reference price itself; this clearly benefits consumers as it allows them to increase their consumption levels.

Government expenditure under RP depends on both $\varepsilon$ and $\theta$. In particular, as the copayment rate $\theta$ increases, thus contributing to lower expenditure, the headline generic price (i.e., the reference price for reimbursement) increases, thus contributing to higher expenditure. However, demand for both the branded and generic pharmaceuticals decreases, thus contributing to lower expenditure. Therefore, the net effect of changes in $\theta$ on government expenditure depends on the magnitudes of these effects, whereas under FPR higher copayment rates unambiguously reduce expenditure. Figure 1 shows that government expenditure is higher for lower values of $\varepsilon$, because the reference price is higher. Government expenditure approaches 0 as the copayment rate $\theta$ tends to 1 and depending on the value of $\varepsilon$ - it can (as in the case of FPR) decrease with $\theta$ (when $\varepsilon<1 / 2$ ) or it can exhibit an increasing segment (for low values of $\theta$ ) and a decreasing segment (for high values of $\theta$ ) in its relationship with $\theta$ (when $\varepsilon \geqslant 1 / 2$ ). This is particularly relevant from a policy point of view, because it suggests that an increase in the copayment rate might not always succeed in reducing government expenditure.

\footnotetext{
${ }^{16}$ Brekke et al. (2011) obtain a different result. They find that headline prices decrease with the copayment rate, but we must be cautious in interpreting this difference. Indeed, their model is different from ours in more than one dimension: they assume unit demand (whilst we assume elastic demand), and they also assume that the market is not fully covered (whilst we assume full market coverage). In our model, under RP, an increase in $\theta$ works to the branded producer's advantage, as this allows it to raise headline prices and profits. By contrast, the generic producer's profits can increase or decrease with $\theta$, and this depends on the particular value of $\varepsilon$. For the generic producer, the net effect on profits depends on the balance between higher headline (and effective) prices and lower demand.

${ }^{17}$ This is also true in the case of $\varepsilon=0$ (inelastic demand), a result similar to that of Brekke et al. (2007).
} 


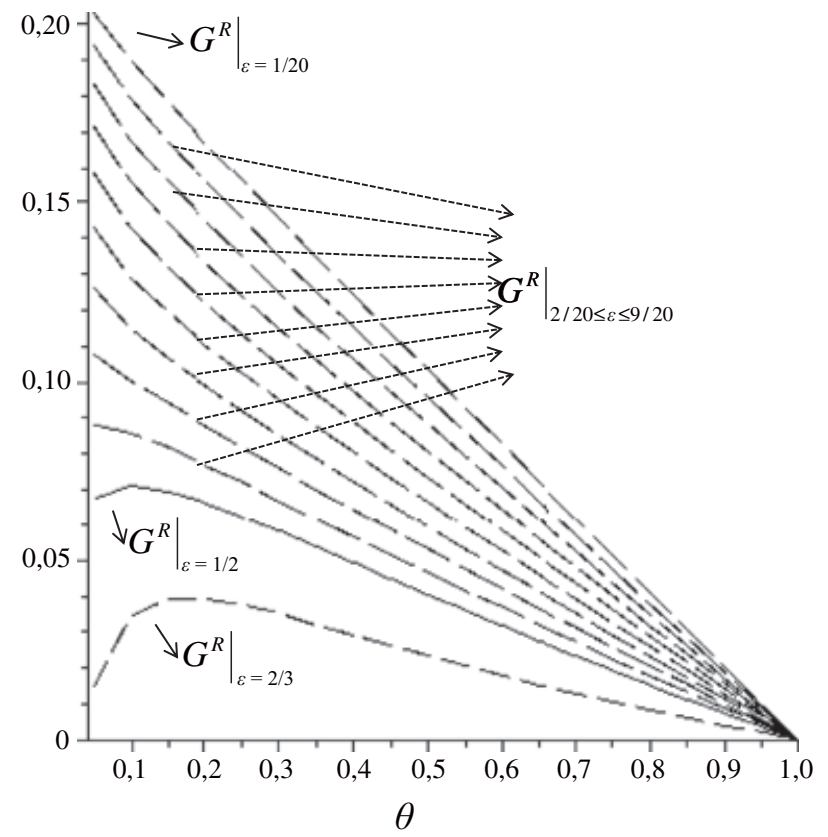

Fig. 1. Government expenditure under RP for various values of $\varepsilon$, with $t=2 / 3$

For a given elasticity, the price differences across schemes become lower as the copayment rate increases. However, for a given copayment rate, higher elasticity values result in lower (absolute) price differences between FPR and RP, but the relative difference (in percentage terms) actually increases. This shows that higher demand elasticities reinforce the merit of RP with regards to FPR, as can be seen in Figure A1 (see the Appendix).

\section{Welfare Analysis}

We now turn our attention to the welfare implications of our results. Following the approach outlined in Section III, we analyze the case of $\varepsilon=1 / 2$; however, simulations show that results are robust to other values of $\varepsilon$ (see the Online Appendix). We focus on social welfare (SW), which is the sum of consumer and producer surplus minus government expenditure with pharmaceuticals. We show the following.

Proposition 3. When $\varepsilon=1 / 2$, social welfare under $R P$ is decreasing with the copayment rate.

Proof: Assuming $\varepsilon=1 / 2$, under an RP scheme social welfare is given by $\left.S W^{R}\right|_{\varepsilon=1 / 2}=\left.C S^{R}\right|_{\varepsilon=1 / 2}+\left.\Pi^{R}\right|_{\varepsilon=1 / 2}-\left.G^{R}\right|_{\varepsilon=1 / 2}$. Using equations (A23), (A24), and (A25), we obtain 


$$
\left.S W^{R}\right|_{\varepsilon=1 / 2}=m-\frac{\left(3 \theta+\theta^{1 / 2}+2 \theta^{3 / 2}\right) t}{2\left(1+2 \theta^{1 / 2}\right)^{2}} .
$$

The derivative of this expression with respect to $\theta$ is unambiguously negative for $\forall \theta \in[0,1]$ :

$$
\frac{\left.\partial S W^{R}\right|_{\varepsilon=1 / 2}}{\partial \theta}=-\frac{\left(1+4 \theta^{1 / 2}+6 \theta+4 \theta^{3 / 2}\right) t}{4 \theta^{1 / 2}\left(1+2 \theta^{1 / 2}\right)^{3}} .
$$

Proposition 4. When $\varepsilon=1 / 2$, social welfare under $R P$ is never lower than under FPR.

Proof: Under FPR, social welfare is given by $\left.S W^{F}\right|_{\varepsilon=1 / 2}=\left.C S^{F}\right|_{\varepsilon=1 / 2}+$ $\left.\Pi^{F}\right|_{\varepsilon=1 / 2}-\left.G^{F}\right|_{\varepsilon=1 / 2}$. Using the expressions in equations (A8) (A9), and (A10) evaluated at $\varepsilon=1 / 2$, we obtain

$$
\left.S W^{F}\right|_{\varepsilon=1 / 2}=m-\frac{t}{3} .
$$

Using this equation, as well as equation (10), the difference between social welfare under the two reimbursement schemes is given by

$$
\left.S W^{F}\right|_{\varepsilon=1 / 2}-\left.S W^{R}\right|_{\varepsilon=1 / 2}=\frac{\left(-2-5 \theta^{1 / 2}+\theta+6 \theta^{3 / 2}\right) t}{6\left(1+2 \theta^{1 / 2}\right)^{2}} .
$$

The denominator is always positive and $t$ is also assumed to be strictly positive. Therefore, the sign of this expression depends on the sign of the non-linear function in the numerator, which is negative for $\forall \theta \in[0,1)$ (in which case $\left.S W^{F}\right|_{\varepsilon=1 / 2}<\left.S W^{R}\right|_{\varepsilon=1 / 2}$ ) and equal to zero when $\theta=1$ (in which case $\left.\left.S W^{F}\right|_{\varepsilon=1 / 2}=\left.S W^{R}\right|_{\varepsilon=1 / 2}\right)$.

In order to understand the rationale underlying Propositions 3 and 4, it is helpful to break down social welfare using only the main components: the location of the indifferent consumer, (effective) prices, and quantities. This yields

$$
S W^{i}=m-\underbrace{\frac{t\left(c^{i}\right)^{2}}{2}}_{\text {transport }}-\frac{\varepsilon}{1-\varepsilon} \underbrace{\left(\hat{p}_{b}^{i} B^{i}+\hat{p}_{g}^{i} G^{i}\right)}_{\text {expenditure }}, \quad i=F, R .
$$

When $\theta=1$, we have already seen that $c^{F}=c^{R}$ and all prices and quantities are equal across reimbursement regimes (i.e., $p_{b}^{F}=p_{b}^{R}, \hat{p}_{b}^{F}=$ $\hat{p}_{b}^{R}, B^{F}=B^{R}$, and $\left.G^{F}=G^{B}\right)$. Therefore, $S W^{F}=S W^{R}$. Under RP, as $\theta$ decreases, Proposition 1 shows that effective prices decrease as well. The equilibrium location of the indifferent consumer $c^{R}$ will also decrease; 
that is, the reduction in the branded (effective) price is more significant than the reduction in the generic (effective) price, and a higher fraction of consumers will purchase the branded pharmaceutical. Therefore, the "transport cost" term in equation (14) becomes smaller and social welfare increases. At the same time, the equilibrium reduction in both effective prices will lead to an increase in the total quantity consumed of both pharmaceuticals, $B^{R}$ and $G^{R}$. The price effect dominates and total consumer pharmaceutical expenditure - the "expenditure" term in equation (14) decreases, thus also increasing social welfare. For low elasticities $(0<\varepsilon<$ $1 / 2$ ) under RP, as $\theta$ decreases, social welfare increases as does generic penetration (see Figure A2 in the Appendix). In addition, for a given $\theta$ and for low elasticities $(0<\varepsilon<1 / 2)$, generic penetration is higher under RP than under FPR. Usually, policies that aim to increase welfare encompass increased generic penetration, which in our model is true for low elasticities. ${ }^{18}$ Note, in particular, the contrast with the case of inelastic demand $(\varepsilon=0)$. In that case, the "expenditure" term in equation (14) is equal to zero, the "transport cost" term does not depend on $\theta$ and social welfare is, therefore, invariant to changes in $\theta$.

In addition, note that social welfare under FPR is constant: neither the "transport cost" term nor the "expenditure" term in equation (14) are sensitive to changes in $\theta$. FPR is a public subsidy to consumers, so that effective prices are lower than headline prices. However, as we have argued earlier, under FPR, it is pharmaceutical firms that effectively profit from this public subsidy, because consumers do not benefit from lower (effective) prices: the mark-up of headline prices (proportional to the reimbursement rate) is fully borne by the government, and hence this transfer is neutral from a social welfare perspective. From a policy point of view, this result clearly indicates the need for pharmaceutical price regulation prior to the adoption of an FPR scheme.

Proposition 4 shows that social welfare under RP is always higher than under FPR (except when $\theta=1$, when they are equal). As $\theta$ becomes lower than one, social welfare under FPR does not change whilst social welfare under RP increases; this is the underlying rationale for Proposition 4. Therefore, from a policy point of view, this confirms the welfare merits of RP when compared with FPR, a result that eluded Brekke et al. (2007) because of their (inelastic demand) modelling assumptions (see

\footnotetext{
${ }^{18}$ Also, the model was constructed with a view towards introducing price-sensitivity in demand. In particular, in the treatment of some health conditions that require prolonged treatments, treatment adherence - which has been shown to depend on patients' copayments - is not full (i.e., copayments might be a hindering factor in the purchase of pharmaceuticals). Under those circumstances, policies that help to reduce effective prices can enhance treatment adherence (effectively increasing consumption), and can ultimately result in higher social welfare. We thank a referee for this interpretation of our results.
} 
Proposition 6 of Brekke et al., 2007). This can be seen when we look at the case of $\varepsilon=0$ (inelastic demand) - we obtain the result of Brekke et al. (2007) that social welfare levels under FPR and RP are equal for any $\theta$.

\section{Discussion and Conclusion}

In this paper, we study the properties of two commonly adopted reimbursement schemes, FPR and RP, in a setting in which consumers' individual demands can be price-elastic. The elastic demand assumption uncovers novel results under RP, as higher copayment rates lead to higher prices. In addition, social welfare under RP is shown to decrease as the copayment rate increases, converging to FPR social welfare levels as the copayment rate approaches one. Therefore, it is a particularly advantageous reimbursement scheme (compared to FPR) for low copayment rates (high reimbursement rates).

On the theoretical side, we note that some of our results deviate from the extant literature. Particularly, our conclusion - that, under RP, prices are increasing in the copayment rate - differs from Brekke et al. (2007) and Gonçalves et al. (2015), who find that prices are independent of the copayment rate, and from Brekke et al. (2011), who conclude that they are decreasing in the same variable. These models are built on different assumptions, and further work seems necessary to reconcile them and to fully characterize the conditions under which each of the possibilities holds.

Our results under $\mathrm{RP}$ are amenable to empirical testing, along the following lines. First, the impact of the copayment rate increases on headline prices is (in absolute terms) decreasing with the elasticity of demand. Second, depending on the elasticity of demand, the copayment rate might not have a monotonic relation with government expenditure. Third, depending on the elasticity of demand, the branded producer's market share can increase or decrease with the copayment rate.

An important question is whether our results hold for conditional direct utility and demand functions different from the ones we consider. Although many alternative set-ups are possible, we have solved the model assuming the specification of Rath and Zao (2001), which yields linear demand functions, and we have obtained similar results (for some parameter values). ${ }^{19}$ It might be worthwhile to extend the analysis to a third possible and, to the best of our knowledge, as yet under-researched, reimbursement scheme: asymmetric fixed percentage reimbursement, through which the reimbursement rates are different for different types of pharmaceuticals,

\footnotetext{
${ }^{19}$ See the Online Appendix for more details.
} 
with generics typically attracting higher reimbursement (lower copayment) rates. ${ }^{20}$ Whilst this type of scheme introduces more complexity in the analysis, it might also have the merit to uncover more intricate details of firms' pricing incentives. Moreover, a consideration of generic competition (as in Ghislandi, 2011) and/or different forms of competition between the branded and generic producer(s) (e.g., Stackelberg or Cournot) might also allow for a better understanding of pricing incentives under each reimbursement scheme. These are likely to be the next steps in our research.

\section{Appendix: Detailed Pricing Calculations and Graphics}

\section{Fixed Percentage Reimbursement}

Under FPR, the effective prices for the branded and generic drugs are given by $\hat{p}_{b}^{F}=\theta p_{b}^{F}$ and $\hat{p}_{g}^{F}=\theta p_{g}^{F}$, respectively. The marginal consumer $c^{F}$, who is indifferent between buying the branded $b$ or generic $g$ pharmaceuticals, is found by solving $\bar{v}_{b}\left(\hat{p}_{b}, m, c\right)=\bar{v}_{g}\left(\hat{p}_{g}, m, c\right)$, where $\bar{v}_{i}(\cdot)$ are the conditional indirect utility functions. This yields

$$
c^{F}=\frac{\theta^{1-\varepsilon}\left[\left(p_{b}^{F}\right)^{1-\varepsilon}-\left(p_{g}^{F}\right)^{1-\varepsilon}\right]}{(1-\varepsilon) t} .
$$

As consumers are assumed to form a unit mass, $c^{F}$ gives us the fraction of consumers purchasing the generic variety and $\left(1-c^{F}\right)$ is the fraction of consumers purchasing the branded pharmaceutical. Consumers in the $\left[0, c^{F}\right]$ interval choose to purchase $\bar{q}_{g}^{F}=1 /\left(\hat{p}_{g}^{F}\right)^{\varepsilon}$ units of the generic drug, whilst consumers in the $\left[c^{F}, 1\right]$ interval choose to purchase $\bar{q}_{b}^{F}=1 /\left(\hat{p}_{b}^{F}\right)^{\varepsilon}$ units of the branded drug $\left(\bar{q}_{i}(\cdot), i \in\{b, g\}\right.$ are the conditional demand functions). Let $\mathbf{p}^{F}=\left(p_{b}^{F}, p_{g}^{F}\right)$ represent the price vector. Using equation (3), the demand functions are thus given by

$$
\begin{aligned}
B\left(\mathbf{p}^{F}\right) & =\bar{q}_{b}^{F}\left(1-c^{F}\right)=\frac{1}{\theta^{\varepsilon}\left(p_{b}^{F}\right)^{\varepsilon}}\left\{1-\frac{\theta^{1-\varepsilon}\left[\left(p_{b}^{F}\right)^{1-\varepsilon}-\left(p_{g}^{F}\right)^{1-\varepsilon}\right]}{(1-\varepsilon) t}\right\} ; \\
G\left(\mathbf{p}^{F}\right) & =\bar{q}_{g}^{F} c^{F}=\frac{1}{\theta^{\varepsilon}\left(p_{g}^{F}\right)^{\varepsilon}} \frac{\theta^{1-\varepsilon}\left[\left(p_{b}^{F}\right)^{1-\varepsilon}-\left(p_{g}^{F}\right)^{1-\varepsilon}\right]}{(1-\varepsilon) t} .
\end{aligned}
$$

The profit functions are given by $\Pi_{b}\left(\mathbf{p}^{F}\right)=p_{b}^{F} B\left(\mathbf{p}^{F}\right)$ and $\Pi_{g}\left(\mathbf{p}^{F}\right)=$ $p_{g}^{F} G\left(\mathbf{p}^{F}\right)$ for the branded and generic producers, respectively. Maximizing

\footnotetext{
${ }^{20}$ This type of scheme has been used, for instance, in Portugal in order to help increase generic penetration at an early development stage.
}

(c) The editors of The Scandinavian Journal of Economics 2016. 
these functions with respect to $p_{b}^{F}$ and $p_{g}^{F}$, we obtain the best-response functions:

$$
\begin{aligned}
& p_{b}^{F}=\left[\frac{(1-\varepsilon) t}{2 \theta^{1-\varepsilon}}+\frac{\left(p_{g}^{F}\right)^{1-\varepsilon}}{2}\right]^{1 /(1-\varepsilon)} \\
& p_{g}^{F}=\frac{p_{b}^{F}}{2^{1 /(1-\varepsilon)}}
\end{aligned}
$$

Thus, we obtain the following Nash equilibrium prices:

$$
\begin{aligned}
& p_{b}^{F}=\left[\frac{2(1-\varepsilon) t}{3 \theta^{1-\varepsilon}}\right]^{1 /(1-\varepsilon)} \\
& p_{g}^{F}=\left[\frac{(1-\varepsilon) t}{3 \theta^{1-\varepsilon}}\right]^{1 /(1-\varepsilon)}
\end{aligned}
$$

Therefore, equilibrium individually consumed quantities are given by

$$
\begin{aligned}
& \bar{q}_{b}^{F}=\left[\frac{3}{2(1-\varepsilon) t}\right]^{\varepsilon /(1-\varepsilon)} \\
& \bar{q}_{g}^{F}=\left[\frac{3}{(1-\varepsilon) t}\right]^{\varepsilon /(1-\varepsilon)}
\end{aligned}
$$

whilst overall quantities sold are given by

$$
\begin{aligned}
B^{F} & =\left(\frac{2}{3}\right)^{(1-2 \varepsilon) /(1-\varepsilon)}\left[\frac{1}{(1-\varepsilon) t}\right]^{\varepsilon /(1-\varepsilon)} ; \\
G^{F} & =\left(\frac{1}{3}\right)^{(1-2 \varepsilon) /(1-\varepsilon)}\left[\frac{1}{(1-\varepsilon) t}\right]^{\varepsilon /(1-\varepsilon)} .
\end{aligned}
$$

At the equilibrium prices, $c^{F}=1 / 3$. This implies that whilst each individual consumer purchases more units of the generic drug than of the branded drug (as can be seen in equation (A6), noting that $\varepsilon<1$ by assumption), the branded drug is sold to a larger fraction of consumers.

In equilibrium, profits are

$$
\begin{aligned}
\Pi_{b}^{F} & =\frac{4}{9} \frac{1}{\theta}(1-\varepsilon) t \\
\Pi_{g}^{F} & =\frac{1}{9} \frac{1}{\theta}(1-\varepsilon) t
\end{aligned}
$$

Consumer surplus $C S$ is given by the sum of the surplus of buying the generic and branded pharmaceuticals (in equilibrium, $c^{F}=1 / 3$ ):

(c) The editors of The Scandinavian Journal of Economics 2016. 


$$
\begin{aligned}
C S^{F}= & \int_{0}^{1 / 3} \bar{v}_{g}\left(\hat{p}_{g}^{F}, m, c\right) d c+\int_{1 / 3}^{1} \bar{v}_{b}\left(\hat{p}_{b}^{F}, m, c\right) d c \\
= & \int_{0}^{1 / 3}\left\{\bar{q}_{h}\left(\hat{p}_{g}^{F}, m\right)-\frac{\varepsilon}{1-\varepsilon}\left[\bar{q}_{g}\left(\hat{p}_{g}^{F}, m\right)\right]^{(\varepsilon-1) / \varepsilon}-t c\right\} d c \\
& +\int_{1 / 3}^{1}\left\{\bar{q}_{h}\left(\hat{p}_{b}^{F}, m\right)-\frac{\varepsilon}{1-\varepsilon}\left[\bar{q}_{b}\left(\hat{p}_{b}^{F}, m\right)\right]^{(\varepsilon-1) / \varepsilon}\right\} d c \\
= & m-\frac{11 t}{18} .
\end{aligned}
$$

Total profits, which are equivalent to total pharmaceutical expenditure, are given by

$$
\Pi^{F}=\Pi_{b}^{F}+\Pi_{g}^{F}=\frac{5}{9} \frac{1}{\theta}(1-\varepsilon) t .
$$

Expenditure on pharmaceuticals by the government (or other third-party payers) is a proportion $(1-\theta)$ of total pharmaceutical expenditure:

$$
G^{F}=(1-\theta) \Pi^{F}=\frac{5}{9} \frac{(1-\theta)}{\theta}(1-\varepsilon) t .
$$

\section{Reference Pricing}

Under an RP scheme, the effective prices for the branded and generic drugs are $\hat{p}_{b}^{R}=p_{b}^{R}-(1-\theta) p_{g}^{R}$ and $\hat{p}_{g}^{R}=\theta p_{g}^{R}$, respectively (see equation (4)), and the marginal consumer $c^{R}$, who is indifferent between buying the branded $(b)$ or generic $(g)$ pharmaceuticals, is again found by solving $\bar{v}_{b}\left(\hat{p}_{b}, m, c\right)=\bar{v}_{g}\left(\hat{p}_{g}, m, c\right)$. In this case, this yields

$$
c^{R}=\frac{\left(\hat{p}_{b}^{R}\right)^{1-\varepsilon}-\left(\hat{p}_{g}^{R}\right)^{1-\varepsilon}}{(1-\varepsilon) t}=\frac{\left[p_{b}^{R}-(1-\theta) p_{g}^{R}\right]^{1-\varepsilon}-\left(\theta p_{g}^{R}\right)^{1-\varepsilon}}{(1-\varepsilon) t} .
$$

Note that assuming constant headline prices $\left(p_{b}^{R}\right.$ and $\left.p_{g}^{R}\right)$ and $\varepsilon=1 / 2$, we obtain

$$
\frac{\left.\partial c^{R}\right|_{\varepsilon=1 / 2}}{\partial \theta}=\frac{p_{g}}{t}\left[\frac{\left(\hat{p}_{g}^{R}\right)^{1 / 2}-\left(\hat{p}_{b}^{R}\right)^{1 / 2}}{\left(\hat{p}_{b}^{R}\right)^{1 / 2}\left(\hat{p}_{g}^{R}\right)^{1 / 2}}\right]<0 .
$$

Similarly to the FPR case, $c^{R}$ gives us the fraction of consumers purchasing the generic drug under RP. Each consumer in the $\left[0, c^{R}\right]$ interval chooses to purchase $\bar{q}_{g}^{R}=1 /\left(\hat{p}_{g}^{R}\right)^{\varepsilon}$ units of the generic drug, whilst consumers in the $\left[c^{R}, 1\right]$ interval choose to purchase $\bar{q}_{b}^{R}=1 /\left(\hat{p}_{b}^{R}\right)^{\varepsilon}$ units of 
the branded drug. Let $\mathbf{p}^{R}=\left(p_{b}^{R}, p_{g}^{R}\right)$ represent the price vector. The demand functions are thus given by $B\left(\mathbf{p}^{R}\right)=\bar{q}_{b}^{R}\left(1-c^{R}\right)$ for the branded drug, and by $G\left(\mathbf{p}^{R}\right)=\bar{q}_{g}^{R} c^{R}$ for the generic drug. The profit functions are given by $\Pi_{b}\left(\mathbf{p}^{R}\right)=p_{b}^{R} B\left(\mathbf{p}^{R}\right)$ and $\Pi_{g}\left(\mathbf{p}^{R}\right)=p_{g}^{R} G\left(\mathbf{p}^{R}\right)$. When we maximize the latter profit function with respect to $p_{g}^{R}$, the first-order condition for the generic producer yields

$$
\begin{aligned}
& \frac{\partial \Pi_{g}\left(\mathbf{p}^{R}\right)}{\partial p_{g}^{R}}=0 \Leftrightarrow \frac{1}{\theta^{\varepsilon}\left(p_{g}^{R}\right)^{\varepsilon} t} \\
& \quad \times\left\{\frac{p_{b}^{R}-2(1-\theta) p_{g}^{R}}{\left[p_{b}^{R}-(1-\theta) p_{g}^{R}\right]^{\varepsilon}}-2 \theta^{1-\varepsilon}\left(p_{g}^{R}\right)^{1-\varepsilon}\right\}=0 .
\end{aligned}
$$

Maximizing the branded producer's profit function with respect to $p_{b}^{R}$ and setting it equal to zero $\left(\partial \Pi_{b}\left(\mathbf{p}^{R}\right) / \partial p_{b}^{R}=0\right)$ results in the following first-order condition:

$$
\begin{aligned}
& \frac{\partial \Pi_{b}\left(\mathbf{p}^{R}\right)}{\partial p_{b}^{R}}=0 \Leftrightarrow \frac{1}{(1-\varepsilon) t\left(\hat{p}_{b}^{R}\right)^{\varepsilon}} \\
& \quad \times\left\{\left[(1-\varepsilon) t+\left(\hat{p}_{g}^{R}\right)^{1-\varepsilon}\right]\left(1-\frac{\varepsilon p_{b}^{R}}{\hat{p}_{b}^{R}}\right)-\frac{\hat{p}_{b}^{R}+p_{b}^{R}-2 \varepsilon p_{b}^{R}}{\left(\hat{p}_{b}^{R}\right)^{\varepsilon}}\right\} .
\end{aligned}
$$

These first-order conditions cannot be solved analytically and, hence, no closed-form solution exists for equilibrium prices, except in the case of $\varepsilon=1 / 2$. When $\varepsilon=1 / 2$, the generic producer's first-order condition (after some simplifying steps) becomes

$$
\begin{aligned}
\left.\frac{\partial \Pi_{g}\left(\mathbf{p}^{R}\right)}{\partial p_{g}^{R}}\right|_{\varepsilon=1 / 2}= & 0 \Leftrightarrow p_{b}^{R}-2(1-\theta) p_{g}^{R}-2 \theta^{1 / 2}\left(p_{g}^{R}\right)^{1 / 2} \\
& \times\left[p_{b}^{R}-(1-\theta) p_{g}^{R}\right]^{1 / 2}=0 .
\end{aligned}
$$

This non-linear equation has two solutions, but one results in $p_{b}^{R}<p_{g}^{R}$ whenever $\theta>1 / 4$. This violates the assumption that the generic drug is the reference pharmaceutical because it is cheaper, and hence the generic producer's best response is assumed to correspond to this equation's other solution:

$$
p_{g}^{R}=\frac{1-\theta^{1 / 2}}{2(1-\theta)} p_{b}^{R}
$$


With $\varepsilon=1 / 2$, the branded producer's first-order condition yields

$$
\begin{aligned}
\left.\frac{\partial \Pi_{b}\left(\mathbf{p}^{R}\right)}{\partial p_{b}^{R}}\right|_{\varepsilon=1 / 2}= & 0 \Leftrightarrow\left\{t p_{b}^{R}-2 t(1-\theta) p_{g}^{R}-4\left[p_{b}^{R}-(1-\theta) p_{g}^{R}\right]^{3 / 2}\right. \\
& \left.+2 \theta^{1 / 2} p_{b}^{R}\left(p_{g}^{R}\right)^{1 / 2}-4 \theta^{1 / 2}(1-\theta)\left(p_{g}^{R}\right)^{3 / 2}\right\} \\
& \times\left\{2 t\left[p_{b}^{R}-(1-\theta) p_{g}^{R}\right]^{3 / 2}\right\}^{-1}=0 .
\end{aligned}
$$

Substituting the generic producer's best-response function (equation (A16)) into this equation, we obtain the branded producer's Nash equilibrium price. Subsequently, we substitute it into equation (A16) and thus we also obtain the generic producer's equilibrium price:

$$
\begin{aligned}
& \left.p_{b}^{R}\right|_{\varepsilon=1 / 2}=\frac{2 \theta\left(1+\theta^{1 / 2}\right)}{4\left(1+2 \theta^{1 / 2}\right)^{2}} t^{2} ; \\
& \left.p_{g}^{R}\right|_{\varepsilon=1 / 2}=\frac{\theta}{4\left(1+2 \theta^{1 / 2}\right)^{2}} t^{2} .
\end{aligned}
$$

Under RP, the equilibrium individually consumed quantities are thus given by

$$
\begin{aligned}
& \left.\bar{q}_{b}^{R}\right|_{\varepsilon=1 / 2}=\frac{2\left(1+2 \theta^{1 / 2}\right)}{\left(\theta+\theta^{1 / 2}\right) t}, \\
& \left.\bar{q}_{g}^{R}\right|_{\varepsilon=1 / 2}=\frac{2\left(1+2 \theta^{1 / 2}\right)}{\theta t},
\end{aligned}
$$

whilst overall quantities sold are given by

$$
\begin{aligned}
& \left.B^{R}\right|_{\varepsilon=1 / 2}=\left.\bar{q}_{b}^{R}\right|_{\varepsilon=1 / 2}\left(1-\left.c^{R}\right|_{\varepsilon=1 / 2}\right)=\frac{2}{\theta^{1 / 2} t}, \\
& \left.G^{R}\right|_{\varepsilon=1 / 2}=\left.\left.\bar{q}_{g}^{R}\right|_{\varepsilon=1 / 2} c^{R}\right|_{\varepsilon=1 / 2}=\frac{2}{\theta^{1 / 2} t} .
\end{aligned}
$$

At the equilibrium prices, $\left.c^{R}\right|_{\varepsilon=1 / 2}=\theta^{1 / 2} /\left(1+2 \theta^{1 / 2}\right) \leqslant 1 / 3, \forall \theta \in[0,1]$ (in particular, $\left.c^{R}\right|_{\varepsilon=1 / 2}$ is an increasing function of $\theta$ ). This implies that, as under the FPR scheme, whilst each individual consumer purchases more units of the generic drug than of the branded drug, the latter is sold to a larger fraction of consumers.

Note that assuming constant headline prices $\left(p_{b}^{R}\right.$ and $\left.p_{g}^{R}\right)$ and $\varepsilon=1 / 2$, we find that

$$
\begin{aligned}
\frac{\left.\partial B^{R}\right|_{\varepsilon=1 / 2}}{\partial \theta} & =\frac{p_{g}^{R}}{2\left(\hat{p}_{b}^{R}\right)^{3 / 2}}+\left(\frac{\hat{p}_{b}^{R}}{\hat{p}_{g}^{R}}\right)^{1 / 2} \frac{p_{g}^{R}}{t\left(\hat{p}_{b}^{R}\right)^{1 / 2}}\left(p_{b}^{R}-p_{g}^{R}\right)>0, \\
\frac{\left.\partial G^{R}\right|_{\varepsilon=1 / 2}}{\partial \theta} & =\frac{1}{t \theta^{2} p_{g}^{R}}\left(\frac{\hat{p}_{g}^{R}}{\hat{p}_{b}^{R}}\right)^{1 / 2}\left(p_{g}^{R}-p_{b}^{R}\right)<0 .
\end{aligned}
$$


Under an RP scheme with $\varepsilon=1 / 2$, equilibrium profits are

$$
\begin{aligned}
& \left.\Pi_{b}^{R}\right|_{\varepsilon=1 / 2}=\frac{\theta^{1 / 2}\left(1+\theta^{1 / 2}\right) t}{\left(1+2 \theta^{1 / 2}\right)^{2}}, \\
& \left.\Pi_{g}^{R}\right|_{\varepsilon=1 / 2}=\frac{\theta^{1 / 2} t}{2\left(1+2 \theta^{1 / 2}\right)^{2}} .
\end{aligned}
$$

Consumer surplus $C S$ is given by the sum of the surplus of buying the generic and branded pharmaceuticals, which for $\varepsilon=1 / 2$ yields (in equilibrium, $\left.\left.c^{R}\right|_{\varepsilon=1 / 2}=\theta^{1 / 2} /\left(1+2 \theta^{1 / 2}\right)\right)$ :

$$
\begin{aligned}
\left.C S^{R}\right|_{\varepsilon=1 / 2}= & \int_{0}^{\left.\theta^{1 / 2 /\left(1+2 \theta^{1 / 2}\right.}\right)} \bar{v}_{g}\left(\left.\hat{p}_{g}^{R}\right|_{\varepsilon=1 / 2}, m, c\right) d c \\
& +\int_{\theta^{1 / 2} /\left(1+2 \theta^{1 / 2}\right)}^{1} \bar{v}_{b}\left(\left.\hat{p}_{b}^{R}\right|_{\varepsilon=1 / 2}, m, c\right) d c \\
= & \int_{0}^{\theta^{1 / 2} /\left(1+2 \theta^{1 / 2}\right)}\left\{\bar{q}_{h}\left(\left.\hat{p}_{g}^{R}\right|_{\varepsilon=1 / 2}, m\right)-\frac{\varepsilon}{1-\varepsilon}\right. \\
& \left.\times\left[\bar{q}_{g}\left(\left.\hat{p}_{g}^{R}\right|_{\varepsilon=1 / 2}, m\right)\right]^{(\varepsilon-1) / \varepsilon}-t c\right\} d c \\
& +\int_{\frac{\theta^{1 / 2}}{1+2 \theta^{1 / 2}}}^{1}\left\{\bar{q}_{h}\left(\left.\hat{p}_{b}^{R}\right|_{\varepsilon=1 / 2}, m\right)-\frac{\varepsilon}{1-\varepsilon}\right. \\
& \left.\times\left[\bar{q}_{b}\left(\left.\hat{p}_{b}^{R}\right|_{\varepsilon=1 / 2}, m\right)\right]^{(\varepsilon-1) / \varepsilon}\right\} d c \\
= & m-\frac{\left(5 \theta+2 \theta^{1 / 2}+4 \theta^{3 / 2}\right) t}{2\left(1+2 \theta^{1 / 2}\right)^{2}} .
\end{aligned}
$$

Note that consumer surplus under RP (with $\varepsilon=1 / 2$ ) is decreasing with $\theta$. This is to be expected because effective prices are increasing with $\theta$ (Proposition 1). When $\varepsilon=1 / 2$, total profits, which are equivalent to total pharmaceutical expenditure, are an increasing function of $\theta$ given by

$$
\left.\Pi^{R}\right|_{\varepsilon=1 / 2}=\left.\Pi_{b}^{R}\right|_{\varepsilon=1 / 2}+\left.\Pi_{g}^{R}\right|_{\varepsilon=1 / 2}=\frac{\left(3 \theta^{1 / 2}+2 \theta\right) t}{2\left(1+2 \theta^{1 / 2}\right)^{2}} .
$$

Expenditure on pharmaceuticals by the government (or other third-party payers) is equivalent to the reimbursement rate $(1-\theta)$ multiplied by the reference price (which we assume to be the generic price $\left.p_{g}^{R}\right|_{\varepsilon=1 / 2}$ ) - giving us the reimbursement amount per unit purchased - further multiplied by 
180 Reference pricing with elastic demand for pharmaceuticals

the total quantity sold. In the case of $\varepsilon=1 / 2$, this leads to

$$
\left.G^{R}\right|_{\varepsilon=1 / 2}=\left.(1-\theta)\left(\left.B^{R}\right|_{\varepsilon=1 / 2}+\left.G^{R}\right|_{\varepsilon=1 / 2}\right) p_{g}^{R}\right|_{\varepsilon=1 / 2}=\frac{\left(\theta^{1 / 2}-\theta^{3 / 2}\right) t}{\left(1+2 \theta^{1 / 2}\right)^{2}} .
$$

\section{Other Relevant Graphics}
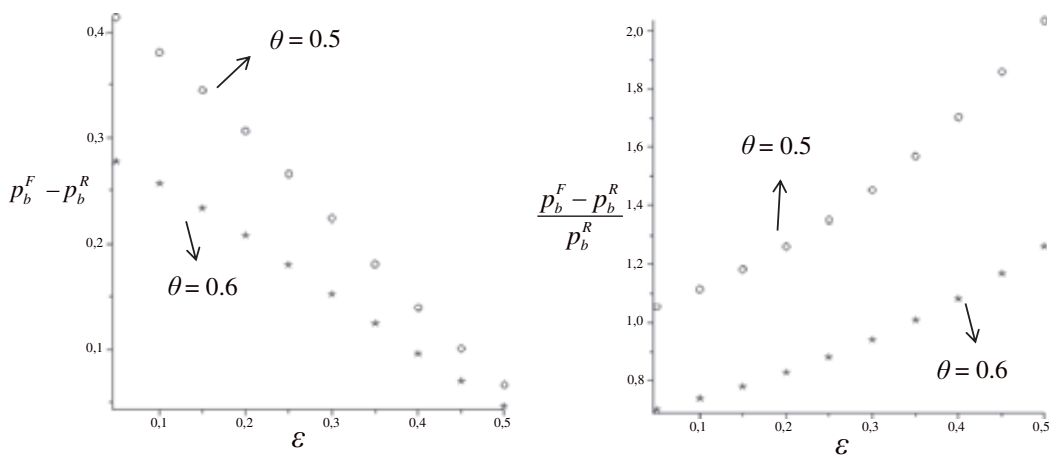

Fig. A1. Absolute (left) and relative (right) price differences $p_{b}$ between FPR and RP for different values of $\varepsilon$ (in the case of $\theta=0.5$ and 0.6 , with $t=2 / 3$ )

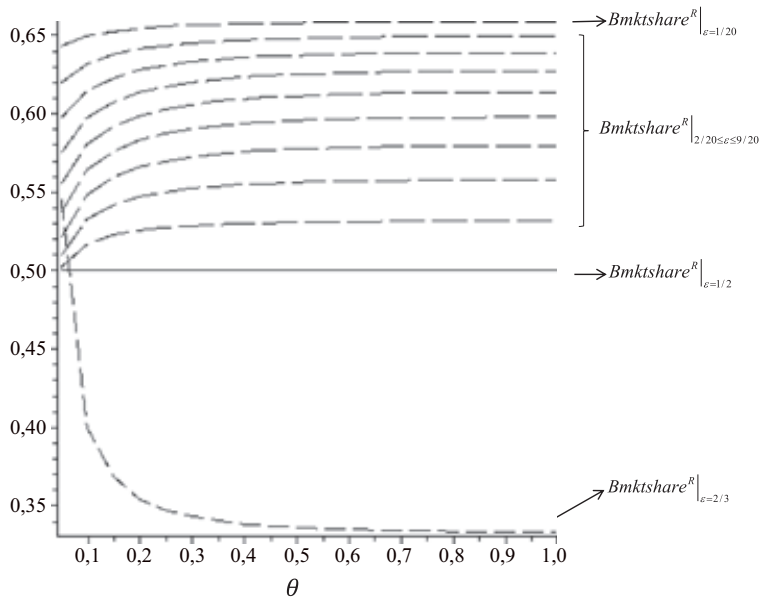

Fig. A2. Branded pharmaceutical market share $B^{R} /\left(B^{R}+G^{R}\right)$ under RP for various values of $\varepsilon$, with $t=2 / 3$

(c) The editors of The Scandinavian Journal of Economics 2016. 


\section{Supporting Information}

The following supporting information can be found in the online version of this article at the publisher's web site.

\section{Online Appendix}

\section{References}

Borgherini, G. (2003), The Bioequivalence and Therapeutic Efficacy of Generic Versus Brand-Name Psychoactive Drugs, Clinical Therapeutics 25, 1578-1592.

Brekke, K., Königbauer, I., and Straume, O. (2007), Reference Pricing of Pharmaceuticals, Journal of Health Economics 26, 613-642.

Brekke, K., Grasdal, A., and Holmås, T. (2009), Regulation and Pricing of Pharmaceuticals: Reference Pricing or Price Cap Regulation?, European Economic Review 53, 170-185.

Brekke, K., Holmås, T., and Straume, O. (2011), Reference Pricing, Competition and Pharmaceutical Expenditure: Theory and Evidence from a Natural Experiment, Journal of Public Economics 95, 624-638.

Eaddy, M., Cook, C., O’Day, K., Burch, S., and Cantrell, R. (2012), How Patient CostSharing Trends Affect Adherence and Outcomes: A Literature Review, Pharmacy and Therapeutics 37, 45-55.

Galizzi, M. M., Ghislandi, S., and Miraldo, M. (2011), Effects of Reference Pricing in Pharmaceutical Markets, PharmacoEconomics 29, 17-33.

Ghislandi, S. (2011), Competition and the Reference Pricing Scheme for Pharmaceuticals, Journal of Health Economics 30, 1137-1149.

Gibson, T., Ozminkowski, R., and Goetzel, R. (2005), The Effects of Prescription Drug Cost Sharing: A Review of the Evidence, American Journal of Managed Care 11, 730740.

Gibson, T., Mark, T., McGuigan, K., Axelsen, K., and Wang, S. (2006), The Effects of Prescription Drug Copayments on Statin Adherence, American Journal of Managed Care 12, 509-517.

Goldman, D., Joyce, G., and Zheng, Y. (2007), Prescription Drug Cost Sharing Associations with Medication and Medical Utilization and Spending and Health, Journal of the American Medical Association 298, 61-69.

Gonçalves, R., Rodrigues, V., and Vasconcelos, H. (2015), Reference Pricing in the Presence of Pseudo-Generics, International Journal of Health Economics and Management 15, 281-305.

Gu, Y. and Wenzel, T. (2009), A Note on the Excess Entry Theorem in Spatial Models with Elastic Demand, International Journal of Industrial Organization 27, 567-571.

Hanemann, W. M. (1984), Discrete/Continuous Models of Consumer Demand, Econometrica $52,541-561$.

Kesselheim, A. S., Misono, A. S., Lee, J. L., Stedman, M. R., Brookhart, M. A., Choudhry, N. K., and Shrank, W. H. (2008), Clinical Equivalence of Generic and Brand-Name Drugs Used in Cardiovascular Disease: A Systematic Review and Meta-Analysis, Journal of the American Medical Association 300, 2514-2526.

Kobayashi, E., Karigome, H., Sakurada, T., Satoh, N., and Ueda, S. (2011), Patients' Attitudes towards Generic Drug Substitution in Japan, Health Policy 99, 60-65.

Lexchin, J. and Grootendorst, P. (2004), Effects of Prescription Drug User Fees on Drug and Health Services Use and on Health Status in Vulnerable Populations: A Systematic Review of the Evidence, International Journal of Health Services 34, 101-122. 
López-Casasnovas, G. and Puig-Junoy, J. (2000), Review of the Literature on Reference Pricing, Health Policy 54, 87-123.

Merino-Castelló, A. (2003), Impact of the Reference Pricing System on the Pharmaceutical Market: A Theoretical Approach, Universität Pompeu Fabra Working Paper 524a.

Miraldo, M. (2009), Reference Pricing and Firms' Pricing Strategies, Journal of Health Economics 28, 176-197.

Quintal, C. and Mendes, P. (2011), Underuse of Generic Medicines in Portugal: An Empirical Study on the Perceptions and Attitudes of Patients and Pharmacists, Health Policy 104, 61-68.

Rath, K. P. and Zhao, G. (2001), Two Stage Equilibrium and Product Choice with Elastic Demand, International Journal of Industrial Organization 19, 1441-1455.

Rodrigues, V., Gonçalves, R., and Vasconcelos, H. (2014), Anticompetitive Impact of PseudoGenerics, Journal of Industry, Competition and Trade 14, 83-98.

Tseng, C., Brook, R., Keeler, E.. Steers, W., and Mangione, C. (2004), Cost-Lowering Strategies Used by Medicare Beneficiaries Who Exceed Drug Benefit Caps and Have a Gap in Drug Coverage, Journal of the American Medical Association 292, 952-960.

First version submitted May 2014;

final version received June 2016. 